\title{
Intermédialités
}

Histoire et théorie des arts, des lettres et des techniques

Intermediality

History and Theory of the Arts, Literature and Technologies

\section{Du documentaire au documontage : Vingt ans après de Sophie Calle}

\section{Johnnie Gratton}

Numéro 7, printemps 2006

Filer (Sophie Calle)

Shadowing (Sophie Calle)

URI : https://id.erudit.org/iderudit/1005523ar

DOI : https://doi.org/10.7202/1005523ar

Aller au sommaire du numéro

Éditeur(s)

Centre de recherche sur l'intermédialité

ISSN

1705-8546 (imprimé)

1920-3136 (numérique)

Découvrir la revue

Citer ce document

Gratton, J. (2006). Du documentaire au documontage : Vingt ans après de Sophie Calle. Intermédialités / Intermediality, (7), 167-179.

https://doi.org/10.7202/1005523ar
Résumé de l'article

En 2001, Sophie Calle se fait suivre pendant une journée par un détective privé. Son objectif : acquérir le rapport et les photos du détective afin d'y ajouter son propre rapport et de compiler ainsi une attestation documentaire hétérogène, à la fois intermédiale et interauctoriale, du projet. C'est à partir de ce dispositif hétérogène que l'on aborde la version du projet publiée au sein de ce numéro spécial. Et c'est en reconnaissance de ce dispositif hétérogène que l'on proposera de caractériser celle-ci comme " documontage », assemblage d'éléments disparates qui ne cesse de contester, au sein d'un exercice documentaire, les protocoles et le régime de vérité propres à l'éthique documentaire. 


\title{
Du documentaire au documontage: Vingt ans après de Sophie Calle
}

\author{
JohnNiE Gratton
}

Vingt ans après. Le titre désigne non seulement un ensemble de photographies et de textes à visée documentaire, mais aussi son antécédent, le projet qui fit l'objet de cette visée documentaire, expérience tout à fait invérifiable, du point de vue du lecteur-regardeur, en dehors de sa représentation documentaire. Cet ensemble - ce «documontage», si je puis dire - se produit aux confins de deux exigences contraires: faire œuvre, «en finir », sans abandonner complètement la dynamique processuelle du projet (travail en cours, valorisation de l'inachevé). En fait, dans le cas de Vingt ans après, Calle aurait trouvé le moyen de résoudre ce paradoxe en destinant son documontage à deux modes de présentation, et donc de réception. Côté exposition, elle produit en 2001, pour la galerie Emmanuel Perrotin, une installation murale, ensemble qu'elle fait remonter au Centre Pompidou en 2003-2004, sans modification aucune, dans le cadre de l'exposition Sophie Calle. M'as-tu vue. D’une présentation à l'autre, le documontage semble revendiquer un statut d'œuvre. Par contre, côté édition, il n'existe pour l'instant aucun équivalent, en termes quantitatifs, de cette œuvre exposée. La version publiée dans le catalogue de l'exposition M'as-tu vue, par exemple, reproduit mot à mot les éléments textuels de l'installation, mais ne garde que le tiers des photos en couleur (onze sur trente-trois)ํ. Ici même, pour ce numéro spécial de la revue Intermédialités, Calle n'a fourni pour accompagner les textes que huit photos, dont quatre qui ne figurent pas dans la sélection opérée pour le livre-catalogue de 2003, mais qui restent plus ou moins visibles sur la petite photo annexe de l'installation originale (fig. 1). Pourtant, à titre de compensation, elle a fourni une composante complètement inédite sous la forme de huit images numérisées liées aux huit photos. Par là, le documontage mis en forme à la suite de cet article

1. Sophie Calle, M'as-tu vue, catalogue de l'exposition, Christine Macel (éd.), Paris, Éditions du Centre Pompidou, Éditions Xavier Barral, 2003, p. 113-122. 
DU DOCUMENTAIRE AU DOCUMONTAGE : VINGT ANS APRÈS DE SOPHIE CALLE

Selon mes instructions, ma mère s'étaít rendue à l"agence * Duluc, Détectives privés *. Elle avait demandé qu'on me prenne en filaturé

L'enquête s'est déroulée le 16 avril 1981 . Vingt ans après, à la requête d'Emmanuel Perrotin, un détective de l'agence Duluc m'a suivic le 16 avril 2001
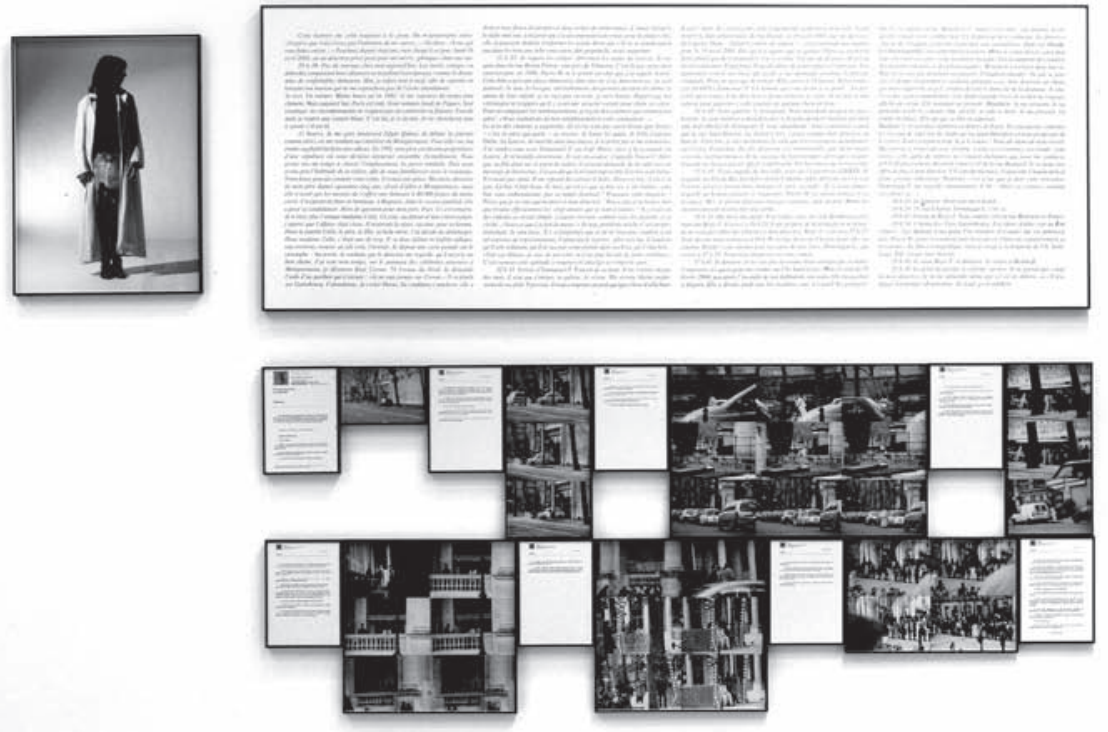

Fig. 1. Sophie Calle, Vingt ans après, vue de l'installation, 2001, Galerie Emmanuel Perrotin, Paris. (c) Sophie Calle/SODRAC 2006. Gracieuseté de la Galerie Emmanuel Perrotin, Paris/Miami. 
présente une version à la fois abrégée et augmentée de l'œuvre-installation : paradoxe percutant qui souligne que, chez Calle, le souci de l'œuvre n'est jamais à confondre avec la fétichisation de l'œuvre.

Vingt ans après. En effet, il s'agit d'un exercice de répétition d'un projet antérieur, La filature (1981), au cours duquel Calle se fit suivre par un détective privé afin de renverser sa pratique, devenue déjà habituelle, de s'attacher à d'autres en les suivant (pratique qu'exemplifie notamment sa Suite vénitienne de 1980). Calle décide, donc, de reprendre La filature comme modèle, source de contraintes - ce dont elle a toujours besoin afin de paramétrer le temps et l'espace, le jeu et l'enjeu, de tel projet. Pour la deuxième fois, elle demande à un intermédiaire d'embaucher un détective de l'agence Duluc pour que celui-ci la prenne en filature pendant une journée prévue et produise une documentation écrite et photographique des activités de sa surveillée. Pour la deuxième fois, elle conduit son «ombre » à travers Paris, en voiture et à pied, vers des lieux de mémoire personnels, de rendez-vous et de visite, lui offrant en filigrane (et finalement en vain) des indications sur son passé, sa famille, ses intérêts, ses préoccupations, son identité. Pour la deuxième fois, elle produit un documontage du projet, un assemblage d'éléments hétérogènes, en ajoutant son propre compte rendu de la journée à la documentation phototextuelle (déjà hétérogène) reçue de son intermédiaire.

L'on peut se demander si Calle avait eu l'idée de réactiver sa «suite parisienne » à partir d'un jeu de mots: donner suite, donner une suite, à son projet antérieur. Hélas, il s'avère que l' «auteur » de cette idée fut un autre, le galeriste Emmanuel Perrotin, qui, à cette époque, cherchait par tous les moyens à recruter l'artiste. Donc, dès le moment de la re-conception du projet, la figure de l'auteur, entendue comme instance singulière et principe unificateur de l'œuvre, commence à se désagréger. D’emblée, la démarche de l'artiste vise une œuvre à venir, au bout du projet, qui portera bien sa seule signature, sans pour autant effacer l'évidence du rôle joué par d'autres prétendants au statut d'«auteur ». La démarche en question innerve, en fait, tous les projets de Calle (comme elle innerve, d'ailleurs, le travail de bien d'autres spécialistes contemporains de l'art du projet ${ }^{2}$ ).

En ce qui concerne Vingt ans après, l'on peut discerner chez l'artiste-auteure au moins trois modes de réquisition, ou de dépendance, d'autres instances auctoriales. D'abord, la commande, car, en disant oui à l'idée du galeriste, Calle

2. Voir Johnnie Gratton et Michael Sheringham (dirs.), The Art of the Project: Projects and Experiments in Modern French Culture, Oxford, New York, Berghahn Books, 2005. 
accepte non seulement de travailler sur commande («J'ai décidé de jouer»), mais de respecter les règles d’un jeu antérieur et extérieur. Facteurs décisifs dans la genèse du projet, l'idée et le modèle sont des germes venus d’ailleurs, des éléments hétérogènes. Ensuite, la collaboration, terme qui décrit le processus de création de la photo liminaire de Vingt ans après, élément de l'installation ainsi que de la version de celle-ci publiée dans le livre-catalogue de 2003. Portrait ou autoportrait? En fait, il s'agit d'un photoportrait en pied dont on peut dire qu'il est tout à la fois «de» Calle (dans la mesure où c'est elle qui pose devant l'objectif et elle qui aurait conçu et commandé le portrait) et «de» Jean-Baptiste Mondino (l'auteur reconnu de la photo). Et enfin la recontextualisation, terme qui recouvre le processus d'appropriation et de conversion des résultats textuels et photographiques d'une mission de surveillance en éléments de ce que Calle appelle «un exercice de représentation». Chez Calle, l'appropriation n'est que peu liée à l'art «appropriationniste ». Il s'agit plutôt, et plus concrètement, du stratagème exécuté au stade préparatif du projet pour obtenir par ruse certains documents indispensables à la réalisation du projet. À l'encontre des complices de l'artiste, le détective contribue au projet, en tant qu'auteur et acteur, sans le savoir. Quant au travail de «conversion» que l'artiste fait subir par la suite à ces documents, il est évident qu’elle réorganise et réoriente les documents qu’elle a réquisitionnés grâce à un travail important (quoique souvent délégué) de mise en forme : étalement des documents, séparation ou regroupement des parties de tel document, encadrement, mise en page, redimensionnement, et ainsi de suite. Ce travail de montage et de monstration ne l'amène pas nécessairement à modifier le «contenu » de ces documents : elle ne veut pas les assimiler. Elle les transpose, geste simple mais efficace en ce qu'il leur assigne un contexte, une fonction, et une réception autres que celles prévues par leur auteur. Si elle modifie, c'est en triant et en abrégeant. L'artiste opère un choix, par exemple, parmi les photos qu'elle reçoit: trente-deux sur au moins quarante-quatre pour son installation, nombre décroissant de onze à huit pour les variantes publiées (voir ci-dessus). Cela dit, d'une version exposée ou imprimée à l'autre, elle se garde d'abréger le relevé graphique du détective. Il s'agirait donc du texte original. Ce qui n'exclut pas qu'elle touche au document original. Car, malgré un semblant d'authenticité issu de l'emploi d'une police démodée donnant l'impression d'un tapuscrit prénumérique, elle modifie la présentation du texte, continue dans la première version imprimée, discontinue et espacée dans l'installation et dans la version publiée au sein de cette revue.

L'hybridation de la dimension auctoriale se recoupe chez Calle, notamment dans le cas de Vingt ans après, avec l'hybridation de la dimension médiale, c'est- 
à-dire avec l'association texte-image, que Régis Durand décrit comme «le premier dispositif multimédia. Qu'il s'agisse de l'ensemble mural ou de ses variantes publiées, la disposition des éléments visuels et textuels témoigne du recours à une forme géométrique simple, le rectangle (forme qui n’a de cesse de s'imposer comme matrice principale de la représentation et comme corollaire actif des processus de production techniques du livre et de l'image photographique), et à un mode d'association simple, la juxtaposition. Simple, mais flexible, car ce mode permet des variations de distance physique entre les éléments convoqués. Là où l'espacement donne lieu à des effets de dissociation (voir les trois «panneaux» de l'installation), le resserrement produit une organisation plus intime, plus interactive, des éléments (voir le panneau composite de l'installation). Évidemment, le resserrement peut aller jusqu'à son degré zéro, le bout-à-bout, le contigu. À noter, cependant, que la mise en contact de deux rectangles, qu'il s'agisse de pages ou de tableaux (au sens large du mot), ne va pas sans tracer une ligne de démarcation: le pli que créent les petits fonds de la double page, le mince interstice entre deux cadres rapprochés. Espaçant sans délier, rapprochant sans unir, le dispositif juxtapositionnel fait appel à un lecteur actif, prêt à faire le va-etvient entre les éléments convoqués, et, partant, à dégager (ou à actionner) le jeu complexe d'énergies conjonctives et disjonctives que lui propose leur cohabitation - d'autant plus que celle-ci s'avère à la fois intermédiale et interauctoriale.

Qu'en est-il donc de ce jeu complexe, tel qu'il ressort de la nouvelle version de Vingt ans après, publiée par Intermédialités? Jusqu'ici, Calle n’a choisi de juxtaposer son propre rapport à celui du détective qu'en les faisant suivre. Cette fois, elle a approuvé l'idée de la maquettiste de la revue d'exploiter le dispositif de la double page de sorte que les deux textes respectifs se déroulent parallèlement l'un à l'autre. D'ailleurs, pour encore mieux encourager le lecteur à faire la navette entre ces deux composantes écrites, l'on a décidé d'opérer de part et d'autre un découpage tel que les correspondances épisodiques entre les deux textes soient mises en regard. Mais il ne s'agit pas uniquement de lire, car ces doubles pages appellent d'emblée la figure intermédiale du lecteur-regardeur. En fait, le débit graphique des deux textes subit deux modes d'interruption : celle de l'insertion de blancs, procédé qui sert à les découper en épisodes, et celle de l'insertion d'images, procédé qui fait alterner texte et «illustration». C'est ici qu'entre en jeu la composante inédite mentionnée ci-dessus, car chacune des huit photos

3. Régis Durand, «Sans commune mesure?», dans Jean-Luc Nancy, Régis Durand, Savine Faupin, Charles Harrison, Alain Fleischer, Jean-Pierre Rehm, Sans commune mesure. Image et texte dans l'art actuel, Paris, Éditions Léo Scheer, 2002, p. 21. 
du détective, c'est-à-dire chaque image numérisée du recto du tirage original, se double désormais d’une image numérisée de son verso, surface normalement cachée, sur laquelle on peut lire, sur un fond de papier Kodak, un certain nombre d'annotations manuscrites systématiquement disposées, mais difficilement lisibles. Ainsi, l'on peut confirmer qu'il s'agit bien de l'affaire Calle («Aff Calle », en haut à gauche). L'on découvre, d'ailleurs, que le détective avait daté et numéroté chaque photo. Élément précieux, le numérotage nous apprend qu'il en avait pris au moins quarante-quatre. Enfin, pour chaque photo, il propose une espèce de légende permettant à son client de mieux repérer les jointures entre ses photos et son texte - et l'on verra que les transcriptions typographiques de ces légendes se situent respectivement au-dessous des photos dont il est question. Chose rare dans l'œuvre de Calle, ces «prises de dos» dépassent le principe de juxtaposition texte-photo en présentant à l'intérieur d'un seul cadre ce que Régis Durand appelle « une matière de pensée dans laquelle texte et image sont d'emblée coprésents ${ }^{\star}$ » : caractérisation de l'intermédialité que l'on pourrait étendre au «cadre » de la double page, tel qu'il a été investi au sein de cette revue. Car la cohabitation dans l'espace de sept doubles pages d'éléments relevant des quatre composantes du documontage (deux textes, deux suites d'images) ne cesse d'encourager le lecteur-regardeur à réaliser - aux deux sens du mot - le potentiel du jeu complexe qu'on lui présente. L'on s'avise, pourtant, d'un aspect inédit - et bien astucieux - de cette mise en forme qui, lui, déborde le dispositif de la double page pour embrasser plutôt celui du recto-verso, car les images représentant les deux côtés de telle épreuve s'adossent l'une à l'autre à travers le papier de telle page. D'où, chose également inédite dans l'œuvre publiée de Calle, la disposition dos à dos des correspondances épisodiques entre les deux textes. L'appel à une mise en relation d'éléments coprésents hétérogènes au sein de la double page se prolonge en une exigence de mise en relation d'éléments décalés, répartis entre recto et verso. À côté des hétérogénéités de l'intermédial et de l'interauctorial, il faut compter désormais avec celle de l'interpaginal.

Que tirer, donc, de ce jeu complexe? En fait, pour ce qui en est du jeu entre les trois composantes issues de l'enquête du détective, l'on arrive assez vite à discerner que celui-ci a tout fait pour annuler le potentiel disjonctif de l'association texte-image en y opposant un travail d'étayage. En disant là où situer les photos dans l'attestation écrite, les annotations affirment leur fonction de preuves par rapport au texte. En montrant l'emplacement du détective à tel moment de son contrôle, les photos valident le présupposé de son attestation, le fait qu’il était

4. Régis Durand, «Sans commune mesure?», p. 22. 
bien là. Il faut que tout s'emboîte. Le stratagème d'étayage, belle illustration des points faibles de la théorie philosophique de la «vérité-cohérence », s'avère nécessaire pour que la déposition globale du détective donne satisfaction au seul destinataire qui compte, son client.

Deuxième facteur de convergence, l'on discerne également entre ces composantes une sorte d'isomorphisme transmédial. Qu'il s'agisse du rapport du détective, de ses prises de vue, ou de ses annotations, l'on se trouve confronté au même degré zéro du médium élu, au même régime du strict nécessaire. Régime pris en charge, d'ailleurs, par ce «nous de majesté », ou «nous d'auteur », qui dépersonnalise l'énonciateur. Rien que des constats simples, désincarnés (sans modalisation affective ou évaluative), et maigres en informations. Ainsi, le texte du rapport épouse la fameuse «pauvreté ontologique» de l'image photographique (contre laquelle le détective preneur d'images ne prend aucune précaution technique ou esthétique), alors qu'en échange, les photos reproduisent la pauvreté expressive du texte. À la grande déception, peut-être, de la suivie, le détectiveécrivain s'interdit de camper un personnage à partir de vaines conjectures. Bien loin des grands héros du polar, il n’aborde pas le visible pour en déduire sa face cachée, sa part d'invisible. De même, le détective preneur d'images ne s'intéresse guère à composer un paysage urbain, une scène, ou un mystère. Aucune trace, d'ailleurs, de voyeurisme dans ses prises de vue. Notre détective suit sans poursuivre, surveille sans s'abandonner au regard masculin (du moins, dans ses dépositions). Chez lui, l'« œil mécanique» n'est pas l’unique apanage de l'appareil. Et plus on se soumet au régime de l'œil mécanique, plus on se refuse tout accès au probatoire. Dans le contexte de l'art du portrait, cela fait longtemps que le visage a cessé d'être la promesse d'une vérité quelconque du sujet. Faute de téléobjectif puissant, toujours obligé de se tenir à bonne distance de son sujet, le détective a peu de chances (et, probablement, peu envie) de réaliser un photoportrait en gros plan de «Madame Sophie CALLE ». Cela dit, ses clichés montrent à l'évidence les efforts du suiveur pour devancer son sujet, afin de pouvoir la photographier de face ou de trois quarts. Rien de plus important pour le détective preneur d'images que la vue frontale ou presque frontale, d'où la fréquence de celle-ci, itération du visage qui redouble celle du nom de Calle dans le rapport du détective. Il s'agit d'un exercice répété de constat et de contrôle, exercice qui cherche à attester l'identité du sujet, à travers ses diverses apparitions photochimiques, en termes de «mêmeté » et non pas d'«ipséité5 ». Il s'agit, donc, moins de

5. Sur cette distinction, voir Paul Ricœur, Soi-même comme un autre, Paris, Éditions du Seuil, coll. «L'ordre philosophique», 1990, p. 13. 
connaître que de reconnaître. Seule exception à la recherche de la frontalité, la photo qui porte la légende «La surveillée au café, arrivée de l'homme en gris », où la suivie n'est montrée que de dos. La prise de dos offre-t-elle la chance d'une vision fugitive, d’une prise «au dépourvu» de l'ipséité? Sans être concluants, les signes de la «mêmeté» du sujet s'accumulent: un coin de visage, la coiffure, la couleur des cheveux, l'écharpe, et surtout cet «imperméable long et blanc» que relève le détective dans le descriptif qu'il consigne au début de son rapport. L'éclat de cette zone de blancheur au centre d'une photo dominée par des zones d'ombre attire inévitablement l'attention du regardeur. Pour mieux la déjouer. Car le manteau, le dos, la prise de dos, pas plus que les annotations consignées aux dos des épreuves, n'offrent finalement aucun accès à un envers quelconque du visible ou du sujet visible. Dans le contexte d'un acte de surveillance où la surveillée sait qu'elle est en train d'être surveillée, cette non-révélation suggère, peut-être, une sorte de contre-vérité générale que viendraient confirmer toutes les photos du détective, à savoir que toute identité visible est une identité de parade.

En se limitant au registre constatif, notre détective construit un univers neutre: sans éclat, sans secret, sans éloquence. Textuellement et photographiquement, ce qu'il «observe » avant tout, ce sont les protocoles modestes mais contraignants qui dirigent tout à la fois son genre d'enquête et le genre de son enquête. Il s'agirait, donc, d'un système de documentation fondamentalement allergique à l'art et à la littérature : bref, anesthétique. Et, pourtant, c'est justement la fascination pour l'anesthétique qui aurait encouragé tant de créateurs, notamment à partir du tournant conceptualiste des années 1960, à adopter la posture en retrait et le style neutre du documentariste. N'oublions pas qu'avant de se faire suivre pour la première fois, c'est Calle qui suivait, et qu'à cette époque elle aimait imiter la pratique du détective et le style de l'enquête policière, quoique non sans bavures. En cela, le détective représente l'une des démarches modèles adoptées et adaptées par Sophie Calle documentariste photo-textuelle : rapprochement (ou détournement) suggéré par le fait que, dans la version du documontage réalisée pour Intermédialités, les photos du détective accompagnent le texte de Calle.

Rien de ceci ne nous empêche de toujours considérer la thèse que ce documontage comporte un élément de contestation - et un essai de reconfiguration - de l'éthique documentaire telle que l'incarne, de façon paradigmatique, voire parodique, le détective. Comme on l'a déjà constaté à plusieurs reprises, Calle ménage une place active au lecteur-regardeur, condition préalable à toute remise en question de l'idéologie documentaire ${ }^{6}$.

6. Sur ce point, voir André Rouillé, La photographie. Entre document et art contemporain, Paris, Éditions Gallimard, coll. «Folio essais», 2005, p. 524-608. 
En même temps, bien sûr, l'artiste se ménage une place active dans ce processus en accolant aux documents du détective la quatrième composante de son documontage: son propre rapport sur la même journée de filature. D’entrée de jeu, il y a donc conjonction, au sens d'une coréférentalité, mais disjonction en ce que l'on passe du point de vue du suiveur à celui de la suivie. Ainsi, Calle nous invite à comparer les documents issus de ces deux points de vue, exercice dialogique d'où son propre texte ne peut certes sortir indemne, mais où il jouera quand même un rôle de repère privilégié en tant que seul document marquant l'intervention de l'artiste. C'est par cette intervention, justement, qu'elle effectue une recontextualisation concrète des documents importés. C'est bien elle qui convoque ces autres documents, elle qui les dispose, elle qui en dispose. Alors que la juxtaposition n'a rien d'hiérarchique en soi, son corollaire, la disposition, produit facilement des effets de prépondérance. L'on remarquera, par exemple, que, dans la version imprimée du documontage dont il est question ici, c'est le texte de Calle qui passe en premier et qui a le dernier mot.

Le rapport de Calle ne s'apparente stylistiquement à celui du détective que par son aspect journal de bord. Coréférentiels, les deux rapports se vouent au même procédé de chronométrage du parcours de la suivie. À d'autres égards, le rapport de Calle déborde de toutes parts celui du détective. Là où le nous du détective délimite un sujet sans singularité(s), le je de Calle inaugure un discours qui se déploie dans l'insouciance du genre, mêlant le compte rendu de l'emploi du temps de la suivie au récit quasi nouvellistique de sa journée et à une écriture intime qui livre, non sans humour et ironie, les ruminations et réflexions personnelles d'une femme qui fait le bilan de sa vie «vingt ans après ». Ce texte vient troubler tous les autres documents dans la mesure où, sur tel aspect de l'emploi du temps de la suivie, il en dit plus long, excédent qui ne cesse de tourner en déficit tout le dicible et tout le visible recueillis par le détective au nom d'une adhésion stricte au réel. Cet échange inégal favorise, donc, une appréhension de tout ce dont se prive la pratique textuelle et photographique, toujours sommaire, du détective. Il est tout à fait compréhensible que le détective n'arrive pas à égaler Calle dans l'art du portrait, vu que la dimension intime du rapport de celle-ci provient du point de vue relativement privilégié d'un sujet pratiquant une écriture de soi. Les normes d'objectivité et d'impersonnalité qui régissent l'éthique documentaire du genre pratiqué par le détective empêchent celui-ci de dire ce qu'il pense ou croit. D'ailleurs, il se limite à un travail de reconnaissance et non pas de connaissance. L'ipséité n’est pas de sa compétence.

Provocatrice de réactions ainsi que de situations, Calle se munit de ruses pour sortir son détective de ce carcan. En résumant sa journée, elle énumère 
trois aspects de son emploi du temps pendant la filature. À l'encontre de ses «errements» (de moments où elle aurait marché au hasard), et de sa «quête de repères» (quête personnelle associée au sentiment d'avoir perdu le fil), ses «rendez-vous forcés» ne sont ni spontanés ni tout à fait personnels. Ils relèvent plutôt d'une sorte de cache-cache, d'une planification stratégique conçue pour éveiller la curiosité du détective: la suivie veut «conduire» son suiveur, lui «montrer» ou «offrir» des indices, le mettre sur la piste. Mais, à ce stade du projet, Calle ne tient les fils que pour être déçue, car son détective n'est pas un déchiffreur. L'échec quasiment total de cette stratégie ne sert qu'à alimenter la mise en cause de l'éthique documentaire. Être prudent, ne pas «fictionner », adhérer au réel, n'est-ce pas encourir le risque de manquer l'essentiel? Se révèle ici la spécificité de la démarche de Calle à l'égard du documentarisme, à savoir que, dans Vingt ans après, elle s'intéresse moins à une mise en cause de l'éthique documentaire en soi, qu'à la façon dont le documentariste assume cette éthique en personne, en tant que personne, en offrant — ou en n'offrant pas — sa propre personne. Inévitablement, la catégorie de la «personne» dépasse la seule figure du scripteur pour comprendre celle de l'enquêteur : que fait-il, où est-il, qui est-il, lors de ses investigations? C'est justement en vue de pouvoir poser de telles questions que Calle recourt aux manœuvres clandestines caractérisant son projet.

Faisons, donc, des allusions «personnelles». Si la stratégie de Calle échoue, c'est par la faute du détective. C'est lui qui échoue, et à plusieurs reprises. Par exemple, dès l'instant où l'artiste (accompagnée de sa mère, «l'autre femme ») entre dans le Centre Pompidou, le détective perd sa trace à cause d'une rupture de «contact visuel ». Échec. Au dire du détective, ce contact est rétabli une heure plus tard, au moment où Calle regagne sa voiture. Malheureusement, un autre «véhicule»s'interpose pour entraver sa poursuite de l'Autobianchi de Calle. Mais, cette fois, la rupture de contact visuel s'avère définitive: le détective ne la reverra plus. Deux échecs de suite. Ou un seul? L'accord des deux acteurs du projet sur le démarrage effectué par Calle vers 16 h 20 recèle, en fait, un différend, car, là où le détective prétend que Calle quitte la rue Bertin Poirée, celle-ci laisse supposer qu'elle quitte une rue de l'île Saint-Louis. À en croire Calle, on dirait que le détective a dû s'éclipser plus tôt et qu'il invente l'incident de la voiture interposée pour se tirer d'affaire. Le départ de la suivie ne se revêt, d'ailleurs, d'aucun étayage visuel (dans les différentes mises en forme du projet, la dernière image vient toujours de la séquence de photos de la file d'attente devant le Centre Pompidou). Ici, l'intermédialité continue à jouer sous la forme d'une absence parlante d'accompagnement photographique. Le «deuxième» échec du détective serait, donc, plutôt un mensonge, une démis- 
sion. Curieusement, c'est dans son compte rendu de cet incident qu'apparaît la seule trace d'une réaction affective de la part de l'énonciateur: « un autre véhicule vient s'interposer [...] et met énormément de temps à se garer [je souligne]». Le mot est bien un signe d'émoi, d'agacement, mais l'affect ne serait qu'un signe de fiction. Chose encore plus curieuse, et non sans rapport avec ce problème de démission, notre détective et celui du projet de vingt ans avant auraient recouru, paraît-il, à la même stratégie mensongère: coïncidence qui rejette le soupçon sur Calle en remettant en question la présupposition que l'histoire globale de ce projet, telle qu'on la reconstitue à partir de l'avant-propos laconique de l'artiste, est une «histoire vraie». Une fois soupçonné dans son intégralité, le projet se retrouve ravalé au rang d'un complot. Mis à l'épreuve du doute, ce projet documentaire se dégrade en performance pseudo-documentaire.

En fait, chez Calle, la reconfiguration négative du documentarisme n'atteint jamais ce point de non-retour, car, jusque dans leur dimension intime, le propre de ses documontages tient à ce qu'ils déploient un «double jeu» capable de faire cohabiter pulsion ironique et souci de véracité7. Témoin, la suite de Vingt ans après. Passé la crise d'un mensonge qui risque de contaminer l'ensemble du projet, Calle s'engage à nous rassurer. En se rendant sur l'île Saint-Louis, elle se dirige vers une histoire «vraie », celle de Bénédicte Vincens; vers une coïncidence «vraie », cette rencontre fortuite et providentielle de la mère de Bénédicte, qui fait dire au compagnon de Calle: «Alors ça t’arrive vraiment ces choses-là... [je souligne]»; et, par là, vers une expérience «vraie», dans la mesure où cette rencontre confère « un sens » à sa journée. En trouvant ce sens, elle connaît «l'instant délicat d’une journée laborieuse», instant décisif (comme diraient le photographe et le nouvelliste) qui lui permettra de quitter enfin la Via Dolorosa qu'a constitué jusqu'ici son parcours. Bien sûr, le détective n'est plus là pour témoigner de cette épiphanie, absence qui prive l'épisode global de toute vérification. Sans l'apport photo-textuel du détective, l'histoire de Calle est une histoire sans endos.

Dans la toute dernière phrase de son rapport, Calle déclare son indifférence à l'égard de son suiveur. Ce désaveu serait-il le signe d'une insouciance plus générale de l'artiste à l'égard de son projet? Est-ce qu'elle démissionne à son tour? Si oui, il ne s'agit nullement de défaitisme. Que Calle cherche à contourner les règles mises en place pour ses propres projets ou celles qui étayent le genre documentaire, c'est moins par une volonté oppositionnelle que, justement, par

7. Thèse avancée, à propos du recueil Des histoires vraies + dix de Calle, dans Johnnie Gratton, «Auto-photo-bio», TDC (Textes et documents pour la classe), Paris, $n^{\circ} 884$, novembre 2004, p. 16-19. 
insouciance, par le moyen dissident de l'insouciance. Tactique « démissionaire», l'insouciance s'inscrit donc dans le projet comme élément actif de contestation de l'éthique documentaire. L’indifférence qui surgit à la fin du rapport de Calle s'explique, par ailleurs, sur le plan narratif. Si la «chute» de ce récit bref révèle que la suivie ne s'intéresse plus à son détective, c'est que, dès l'avènement de son instant décisif, elle est dorénavant occupée à autre chose, par autre chose. Il s'agit, bien entendu, de "la disparue de l'île Saint-Louis ${ }^{8}$ », Bénédicte Vincens. Ce tournant se résume en une phrase d'une simplicité trompeuse : Elle occupe mon histoire. " Qu'il s'agisse de l'histoire d'une journée de filature ou de l'histoire d'une vie, la disparue s'y installe pour passer le témoin - et le devoir de témoignage - à l'artiste qu'elle admirait jusqu'à vouloir vivre comme elle.

«Passage de témoin ». C'est l'expression que Calle emploie pour indiquer la fonction d'une des rencontres qu'elle a organisées à l'avance, celle de sa mère et de son nouveau galeriste, les instigateurs respectifs des deux projets de filature. L'expression est bien choisie, car, en tant que faux clients agissant au nom de Calle, ces deux complices avaient déjà effectué eux-mêmes un passage de témoin. Ceci, d'ailleurs, afin qu'à son tour un détective prenne le relais en endossant sans le savoir le rôle de partenaire dans l'exécution du projet combiné par l'artiste. Ces passages de témoin relèvent, en fait, de tout un travail de déplacement et de redistribution, voué à la production d'une nouvelle figure de l'art documentaire. Dès le début du projet, par exemple, l'idée de documenter une filature ne sert que de prétexte à celle d'accomplir un «exercice de représentation ». Exercice, d'ailleurs, qui prend pour sujet l'auteure du projet, la figure même du documentariste. L'on s'avise, à cet égard, que ce n'est qu'après avoir été invitée à contribuer à l'exposition «Autoportraits photographiques, 1898-1981 » (au Centre Pompidou) que Calle a réalisé son premier projet de filature. C'est dans ce but que l'auteure-documentariste en appelle au principe du passage de témoin pour découper l'espace et le temps de la représentation de soi, pour déléguer à un autre l'acte de représentation de soi (qui devient, par là, représentation d’elle-même). La délégation: "procédure de transfert de compétence qui [...]

8. Calle reprend ici le titre de l'article de Marc Weizmann, paru en 2000 dans Les Inrockuptibles. Les deux premières pages de cet article fournissent l'un des éléments du documontage Une jeune femme disparaît (2003), présenté comme installation dans l'exposition M'as-tu vue et comme ouvrage photo-textuel dans le catalogue de l'expo. Voir Sophie Calle, M'as-tu vue, p. 125-136. À chaque fois, ce documontage suit celui de Vingt ans après. 
confère au sujet concerné une certaine marge d'autonomie ${ }^{9}$ ». Point essentiel, ni le fait de s'approprier les documents du détective, ni celui de les exposer à une critique quelconque, ne récusent leur marge d'autonomie. Passer le témoin, et le travail de témoignage, à un délégué, n'aura de valeur que si l'on respecte la compétence de celui-ci en tant qu'exécutant d'une manière de faire, d'une manière de faire voir, et d'une manière d'écrire autres que les siennes - ne serait-ce que du fait que, chez le détective, il s'agit de manières «professionnelles », sans rapport avec l'ethos, la manière d'être, de l'individu. Chez Calle, par contre, tout part d'une mise en rapport de l'art avec la vie, d'une certaine manière de faire avec une certaine manière d'être. Et l'ethos de Calle exige, justement, que tout exercice de représentation de soi comporte un élément de mise en scène de soi par l'autre: exigence sans doute issue de la pensée photographique, où l'autre (le délégué), c'est l'appareil, d'autant que celui-ci déclenche «l'avènement de moimême comme autre ${ }^{10}$ ». Ces passages, ces déplacements, rendus concrets dans l'assemblage photo-textuel présenté ci-après, provoquent la reconfiguration du documentaire en «documontage».

9. Voir l'article «Délégation », dans Algirdas Julius Greimas et Joseph Courtès, Sémiotique. Dictionnaire raisonné de la théorie du langage, Paris, Éditions Hachette, coll. «Langue Linguistique Communication», 1979, tome 1, p. 87.

10. Roland Barthes, La chambre claire. Note sur la photographie, dans (Euvres complètes, Éric Marty (éd.), Paris, Éditions du Seuil, 1995 [1980], tome 3, p. 1116. 